\section{Death after a Flu Shot: A Viewpoint}

\author{
Michael S Sinyakov* \\ The Mina \& Everard Goodman Faculty of Life Sciences, Bar-llan \\ University, Ramat-Gan 52900, Israel
}

\begin{abstract}
Current influenza-vaccine policy is aimed at universal vaccination. This approach disregards the differences in anti-influenza immunity among vaccinees prior to vaccination (pre-existing immunity/PEI) and might contribute to the low efficiency of current vaccines and, in extreme cases, fatal vaccination outcomes. A novel concept that introduces PEI as a stratification criterion and a fitness index of vaccinees prior to vaccination is suggested.
\end{abstract}

Keywords: Antibodies; Influenza; Pre-existing immunity; Vaccination

These reflections have emerged following reports of the death of elderly people in Italy last winter shortly after influenza vaccination. This was not the first case of this kind, and similar tragic events have been previously reported in other countries as well. Obviously, many more cases of severe deterioration of existing health conditions post-vaccination remain behind the newspaper headlines. Reactions to these cases vary. Some silently ignore them and pretend that nothing has happened, while others panic and abandon the idea of getting vaccinated. Vaccine manufacturers usually deny any kind of causal relationship between the vaccines and the death of vaccinees. Some authors refer to the number of deaths occurring every day shortly after vaccine administration as pure statistical chance and recommend rethinking pharmacovigilance regulations regarding vaccines so as to avoid outbreaks of generalized panic that compromise immunization campaigns - in other words, they call for the censorship of vaccination reports [1]. Regrettably, none of these reactions can claim to be absolutely true and change the situation. An alternative, more responsible approach is to consider these deaths as warning signs, to admit that there really is a vaccination-related problem, and to try to answer two questions: why does it happen and if it can be prevented in the future?

Influenza-associated annual mortality and morbidity rates (with worldwide estimates of 250,000 to 500,000 deaths and 3 to

*Corresponding author: Michael S Sinyakov, The Mina \& Everard Goodman Faculty of Life Sciences, Bar-Ilan University, Ramat-Gan 52900, Israel, Tel: +972 35318205; E-mail: sinyakov@biu.ac.il

Citation: Sinyakov MS (2015) Death after a flu shot: A viewpoint: A Mini-Review. J Infect Non Infect Dis 1: 010.

Received: September 08, 2015; Accepted: December 18, 2015; Published: December 31, 2015
5 million serious cases of illness), along with three devastating pandemic outbreaks that occurred in the 20th century (Spanish flu in 1918/19 with 40-50 million deaths, Asian flu in 1957 with 2 million deaths, and Hong Kong flu in 1968 with 1 million deaths) render the causal influenza virus a real life threat. Vaccination against influenza is the keystone in the attempt to cope with this threat. Basic principles of vaccination have proven themselves over the years: vaccines have been an effective tool in the prevention, regional elimination, and almost total worldwide eradication of a number of life-threatening diseases, and vaccinology as an applied branch of immunology has taken its rightful place in modern medicine. Bearing this in mind, it seems reasonable to suggest that the Achilles heel of influenza vaccinology is the current practice of vaccination. What is wrong with it?

Influenza viruses demonstrate an unusually high rate of mutational changes and genetic reassortments that underlie antigenic drift and shift, respectively, and give rise to newly emerging virus strains, each with a different antigenic structure. The virus regularly outstrips our attempts to get it under control; we can only make predictions as to what virus formulation is going to emerge, and our mistakes in prediction lead to mismatches between vaccine strains and actually circulating strains, inevitably resulting in lower efficiency of the vaccines employed. The conclusion of one of the most rigorous meta-analyses of efficacy and effectiveness of flu vaccines does not sound reassuring: "evidence for consistent high-level protection is elusive for the present generation of vaccines, especially in individuals at risk of medical complications or those aged 65 years or older" [2]. To paraphrase a famous Murphy's Law, Peter Patriarca ironically remarked at the open session of FDA Advisory Committee in July 2001: "with influenza being a very unpredictable agent, in and of itself, anything can and will go wrong". In our attempt to combat the flu virus, we chase an elusive shadow that is always ahead of us.

Nevertheless, with all its imperfections, vaccination still remains the major tool in the confrontation to the virus. Governments in developed countries make huge investments in the manufacture of influenza vaccines and provide major support for influenza vaccination programs. Out of more than 500 human infectious diseases, routine vaccination of certain population groups in the USA is recommended for 17 of them, and only one of these - influenza - is ranked as a must annual universal vaccination for all ages [3]. Moreover, the vaccine manufacturers are outside the jurisdiction of the law. A person who gets seriously injured post-vaccination is not eligible to sue the vaccine manufacturer but rather should turn to a government office (i.e., the US Department of Health and Human Services/Health Resources and Service Administration), and apply for the Vaccine Injury Compensation Program. Influenza vaccination programs imply a universal vaccination in the USA and Canada, and the selective vaccination of high-risk groups in Europe, where there is a distinct tendency towards the same universal vaccination [4]. In other words, influenza vaccination programs aimed at maximal vaccination coverage as well as flu vaccine manufacturers are given top priority. So, where is the flaw?

Even a quick glance at the current situation in influenza vaccinology reveals an amazing paradox: along with ongoing, active 
attempts to develop improved vaccines and mass media propaganda of universal vaccination, people who should benefit from these vaccines are considered to be inert, homogeneous subjects equally suitable for vaccination; at best, they are divided into high-risk groups selected by age, specific conditions of health, or occupational status. I argue that this approach, which disregards the differences among the vaccinees with regard to their anti-influenza immunity existing prior to vaccination (PEI), might largely contribute to the problem.

Due to an extraordinary mutability of the virus, the individual anti-influenza PEI acquired and accumulated in each person's lifespan as a result of exposure to natural viruses and/or flu vaccines greatly varies, and this interpersonal PEI difference largely determines the great variability in vaccination outcome. The role of PEI (and pre-existing antibodies/PEA in particular) as a factor influencing immune response to vaccines, is widely recognized [5-9], and multiple attempts have been made to correct the post-vaccination results for the PEA titers, i.e., PEI is considered a confounding factor that interferes with the evaluation of vaccine efficiency $[10,11]$.

However, PEI is much more than that and represents a basic, highly variable, individual feature of vaccinees directly involved in formulation of the immune response. Diversity is the basis of evolution, including that of viruses and humans, which intrinsically influence each other. This co-evolutionary arms race between pathogenic viruses and their human hosts is one of the most important driving forces in human molecular evolution [12]. The individual diversity of PEI is reflected in the high variability of immune responses, and represents a fundamental feature of the adaptive immune system, with each individual owning a personal adaptive immune repertoire [13]. It is 'the danger of homogeneity' that prevents evolution, and it is a beneficial diversity that favors the emergence and consolidation of evolutionary qualities necessary for survival, including resistance to infectious diseases [14].

Results obtained in our laboratory definitely indicate the decisive role of PEI in the immune response and the importance of PEI as a selection criterion for vaccination. Preliminary separation of the subjects to be immunized and highly heterogeneous with respect to their relevant PEA into homogeneous PEA groups, proved to be essential in order to get meaningful and reproducible post-vaccination results. The relevant PEA and adaptive post-vaccination antibodies manifested negative correlation. Only low PEA subjects were capable of developing an adequate adaptive immune response whereas it made no sense to immunize high PEA carriers that did not produce adaptive antibodies [15]. Moreover, the immunization of high PEA subjects revealed a dangerous trend of $P E A$ reduction at the first stage of vaccination due to the consumption of the PEA and their drastic decrease [16], which means that inadequate vaccination may deprive PEA without producing adaptive antibodies. Therefore, the level of PEA must be taken into consideration prior to vaccination [17].

Based on the above considerations, ignorance of PEI by the practice of universal vaccination may be directly related to the low efficiency of current vaccines and their potential danger to certain recipients, particularly some of the elderly. This frailest cohort is usually considered 'immunosenescent', i.e., possessing age-related impaired immunity, both innate and adaptive, which makes them incapable of developing an adequate immune response to vaccination. Nevertheless, during their long lives, many of the elderly have had multiple encounters with natural flu viruses as well as exposure to flu vaccines. Each of these events left an imprint in their immune memory; they eventually acquired a wide range of influenza-specific PEA, which might be sufficient to ensure cross-protective immunity to at least some of the newly emerging virus strains. One work reported that $67 \%$ of elderly subjects had protective antibodies against relevant A and B strains prior to vaccination [18].

That means, in fact, that the elderly are often high PEA carriers and do not need vaccination. Let us remember that it was the elderly who proved to be best protected in the deadly Spanish flu pandemic and the 2009 pandemic, owing to their PEI potential. By ignoring diversity, considering the entire population or high-risk groups to be immunologically homogeneous, and offering flu vaccines 'over the counter' [19], we are making a serious mistake, behaving like a bull in a china shop and exposing many people who do not need vaccination to unnecessary risk. We must realize that we are dealing with a multifaceted problem, and we only see the tip of the iceberg.

Considering flu vaccines and vaccinees to be equally active, rightful players in vaccination, there are four options of their interaction. It seems reasonable to suggest that only one of these would be beneficial for the vaccinees - an adequate vaccine and eligible recipient. The other three options would be useless at best. At worst (unsuitable vaccine and unsuitable recipient), vaccination may result in the collapse of immunity, severe exacerbation of existing health conditions, and rapid development of fatal complications.

The above considerations do not call for utopic 'personal' flu vaccines, but they do emphasize the critical importance of evaluating individual PEI status prior to vaccination, and using this parameter as a novel stratification/fitness index. This index should be thoroughly refined, and key parameters of pre-existing humoral and cellular immunity having the greatest impact on the immune response to vaccination should be extensively examined. Novel national infrastructures, including banks of regularly updated personal serum and medical histories should be created, and novel methodology should be developed to ensure fast computerized individual evaluations, which is critical in a pandemic period.

This approach will inevitably cause criticism as complicating an already complex problem. However, it may help break the deadlock of the current policy of influenza vaccination, maintain human health and save lives. "The race is on, the stakes are high, and the world is nervously watching" [20].

\section{References}

1. Signorelli C, Odone A, Conversano M, Bonanni $P$ (2015) Deaths after Fluad flu vaccine and the epidemic of panic in Italy. BMJ 350: h116.

2. Osterholm MT, Kelley NS, Sommer A, Belongia EA (2012) Efficacy and effectiveness of influenza vaccines: a systematic review and meta-analysis. Lancet Infect Dis 12: 36-44.

3. Osterholm MT, Kelley NS, Manske JL, Ballering KS, Leighton TR, et al. (2012) The Compelling Need for Game-Changing Influenza Vaccines: An Analysis of the Influenza Vaccine Enterprise and Recommendations for the Future. Center for Infectious Disease Research and Policy (CIDRAP) Report, University of Minnesota, USA.

4. Odone A, Fara GM, Giammaco G, Blangiardi F, Signorelli C (2015) The future of immunization policies in Italy and in the European Union: The Declaration of Erice. Hum Vaccin Immunother 11: 1268-1271.

5. Andrews SF, Kaur K, Pauli NT, Huang M, Huang Y, et al. (2015) High preexisting serological antibody levels correlate with diversification of the influenza vaccine response. J Virol 89: 3308-3317. 
6. Fonville JM, Wilks SH, James SL, Fox A, Ventresca M, et al. (2014) Antibody landscapes after influenza virus infection or vaccination. Science 346: $996-$ 1000 .

7. Reber AJ, Kim JH, Biber R, Talbot HK, Coleman LA, et al. (2015) Pre-existing immunity, more than aging, influences influenza vaccine responses. Open Forum Infect Dis 2: ofv052.

8. Seidman JC, Richard SA, Viboud C, Miller MA (2012) Quantitative review of antibody response to inactivated seasonal influenza vaccines. Influenza Other Respir Viruses 6: 52-62.

9. Talbot HK, Coleman LA, Zhu Y, Spencer S, Thompson M, et al. (2015) Factors associated with maintenance of antibody responses to influenza vaccine in older, community-dwelling adults. BMC Infect Dis 15:195.

10. Beyer WE, Palache AM, Lüchters G, Nauta J, Osterhaus AD (2004) Seroprotection rate, mean fold increase, seroconversion rate: which parameter adequately expresses seroresponse to influenza vaccination? Virus Res 103: 125-132.

11. Voth DW, Feldman HA, Steinschneider A (1966) Comparative responses of elderly persons to aqueous and depot influenza vaccines. Arch Environ Health 13: 576-585.

12. Worobey M, Bjork A, Wertheim JO (2007) Point, counterpoint: The evolution of pathogenic viruses and their human hosts. Annu Rev Ecol Evol Syst 38 : 515-540.
13. Muraille E (2014) Generation of individual diversity: A too neglected fundamental property of adaptive immune system. Front Immunol 5: 208.

14. Haldane JBS (1949) Disease and evolution. La Ricerca Scientifica 19: 1-11.

15. Sinyakov MS, Dror M, Zhevelev HM, Margel S, Avtalion RR (2002) Natural antibodies and their significance in active immunization and protection against a defined pathogen in fish. Vaccine 20: 3668-3674.

16. Sinyakov MS, Dror M, Lublin-Tennenbaum T, Salzberg S, Margel S, et al. (2006) Nano- and microparticles as adjuvants in vaccine design: Success and failure is related to host natural antibodies. Vaccine 24: 6534-6541.

17. Sinyakov MS, Avtalion RR (2009) Vaccines and natural antibodies: A link to be considered. Vaccine 27: 1985-1986.

18. Loebermann M, Voss U, Meyer S, Bosse D, Fritzsche C, et al. (2013) Clinical trial to evaluate the safety and immunogenicity of a trivalent surface antigen seasonal influenza vaccine produced in mammalian cell culture and administered to young and elderly adults with and without $\mathrm{A}(\mathrm{H} 1 \mathrm{~N} 1)$ pre-vaccination. PLoS One 8: e70866.

19. Poland GA, Tosh PK (2012) New influenza vaccines: Promises, perils, and pitfalls. Vaccine 30: $983-984$.

20. Taubenberger JK, Morens DM (2006) Influenza revisited. Emerg Infect Dis 12: $1-2$. 